\title{
Investigation of an amorphous silicon EPID for measurement and quality assurance of enhanced dynamic wedge
}

\author{
Peter B Greer $^{1,2}$ and Michael P Barnes ${ }^{1,2}$ \\ 1. Radiation Oncology Department, Newcastle Mater Hospital, Newcastle, New South Wales \\ 2298, Australia \\ 2. Physics and Mathematical Physics, University of Newcastle, Newcastle, New South Wales \\ 2298, Australia \\ Email: peter.greer@mater.health.nsw.gov.au
}

\begin{abstract}
This work assessed the dosimetric performance of an amorphous silicon electronic portal imaging device (EPID) for measurement and quality assurance of enhanced dynamic wedge (EDW) profile and wedge factor. EPID measurements of EDW profiles were corrected for pixel sensitivity variation and spectral over-response relative to ion-chamber and compared to ion-chamber and diode-array measurements. The dependence of EPID measurements on wedge direction and source to EPID distance was assessed. The long-term stability was investigated by weekly measurement of EDW profiles and wedge factors over a seven month interval. An empirical correction method was developed to improve EPID profile agreement with diode-array measurements. The EPID profiles differed from conventional measurements by up to $5 \%$. Backscatter from the EPID housing was also found to affect measurements by up to $4 \%$, resulting in changes in EPID measured profile with the direction of the moving jaw and source to EPID distance. EPID profile measurements varied by a maximum of $0.3 \%$ (1 SD) within the umbra, and wedge factors varied by $0.3 \%$ ( $1 \mathrm{SD}$ ) over the seven month interval. The correction function improved agreement between EPID and diode-array to within $2 \%$ for all wedge angles and energies. Due to the ease of use and reproducibility of the EPID measured EDW profiles the device is highly suited to regular measurement of EDW.
\end{abstract}

Short title: Investigation of an a-Si EPID for quality assurance of enhanced dynamic wedge 


\section{Introduction}

The Varian (Palo Alto, USA) enhanced dynamic wedge (EDW) is produced by sweeping one of the collimator jaws during the treatment delivery and modifying the accelerator dose-rate during the sweep. Measurement of the beam profile of the enhanced dynamic wedge is essential as part of linear accelerator acceptance and treatment planning system commissioning. These measurements ensure that the EDW wedge angles meet specifications and that the treatment planning system accurately models the EDW profile. This must be followed by regular quality assurance (QA) of the EDW profile and wedge factor to ensure consistency of the wedged delivery.

These measurements require line-detector or two-dimensional dosimetry systems such as ionchamber arrays, film, or diode-arrays. The set-up and measurement of profiles with these can be time consuming. The arrays usually have coarse resolution, while the film measurements must be digitized and calibrated to dose. Amorphous silicon electronic portal imaging systems (EPIDs) present an alternative for measurement of EDW profile. There is virtually no set-up time involved, and the measurements obtained have high resolution and a full twodimensional record of the beam is obtained. The response of the EPIDs is linear (Munro and Bouius 1998, El Mohri et al 1999, McCurdy et al 2001, Greer and Popescu 2003) and central axis reproducibility measurements have been promising (van Esch et al 2004, Louwe et al 2004). Other attractive features are that the images can be stored in the image database, and potentially analyzed immediately at the linear accelerator.

However, accurate dosimetric measurements with these devices can not be considered to be without potential problems. The flood-field correction profile removes the beam profile from the EPID image, and the profile must be restored using a beam profile correction matrix (Warkentin et al 2003, van Esch et al 2004). Alternatively the images can be corrected for pixel sensitivity and over-response relative to ion-chamber to retain the beam profile information in the image (Greer 2005). The scattering properties of the EPID are different from the equivalent depth in water (Greer and Popescu 2003, Chang and Ling 2003), which affects beam profile measurements. Methods to account for this have included deconvolution/convolution techniques to convert the EPID image to a dose to water (Warkentin et al 2003) and EPID prediction models to model the EPID profile (van Esch et al 2004, Siebers et al 2004). The EPIDs also exhibit an energy dependent response due to the high atomic number phosphor layer (McCurdy et al 2001, Kirkby and Sloboda 2005b, Greer 2005). Backscatter from the EPID positioning arm can also contribute to the measured signal non-uniformly (Ko et al 2004). While the flood-field correction contains backscattered signal 
and will effectively remove this from an EPID image acquired with the same field size, for smaller fields that irradiate only part of the EPID positioning arm, the backscatter to a pixel is reduced, introducing artifacts.

The use of a-Si EPIDs for beam quality assurance has been the result of some investigations. El-Mohri et al (1999) compared their prototype EPID with ion-chamber measurements for a $60^{\circ}$ physical wedge. They found excellent agreement when the EPID was operated in direct detection mode (i.e. without the scintillating phosphor present). Grein et al (2002) compared EPID to film for a $60^{\circ}$ physical wedge at 6 and $18 \mathrm{MV}$. They found that the EPID profile was lower near the field edges, or more rounded than the film measurements. The differential energy response of the EPID relative to ion-chamber will affect EPID measurements of physical wedges (Kirkby and Sloboda 2005). Budgell et al (2005) investigated an a-Si EPID for verification of step and shoot intensity modulated radiation therapy (IMRT) verification. They concluded that the device was not suitable for low dose dosimetric QA measurements due to lag/ghosting effects.

The aim of this work was to investigate the performance of an amorphous silicon EPID for measurement and regular quality assurance of EDW profiles and wedge factors. EPID measurements of EDW profiles and wedge-factors were compared to conventional ionchamber and diode-array measurements. The long-term stability of the EPID measurements was investigated by weekly measurement using a reference setup over a seven month interval. Methods to correct EPID measurements to profiles measured with conventional detectors were developed.

\section{Methods and Materials}

\subsection{EPID EDW Profile measurement}

EPID EDW profile measurements were compared to diode-array profiles. A $20 \times 20 \mathrm{~cm}^{2}$ EDW field with wedge angles of $60^{\circ}$ and $30^{\circ}$ and a $10 \times 10 \mathrm{~cm}^{2}$ field with $60^{\circ}$ angle were delivered with 6 and $18 \mathrm{MV}$ nominal energies from a linear accelerator (Varian, Palo Alto, USA). The accelerator uses the upper (Y) jaws to deliver the profile by sweeping one of the Y-jaws with the other jaw fixed. The wedge can be delivered with either the Y1 jaw sweeping (Y1-in wedge) or the Y2 jaw sweeping (Y2-out wedge). With the collimator positioned at the zero angle, the EDW wedge direction is in the inplane direction, with the Y1 jaw sweeping from the couch (target) toward the gantry (gun). The EDW profile was measured with a diode-array (Profiler, Sun-Nuclear, Melbourne, USA) to provide a benchmark measurement. This consists of a linear array of diode detectors of $0.8 \mathrm{~mm}$ detector size spaced at $5 \mathrm{~mm}$. The array was 
positioned at $100 \mathrm{~cm}$ source-to-surface distance with $1.5 \mathrm{~cm}$ and $3.0 \mathrm{~cm}$ solid water buildup for the 6 and $18 \mathrm{MV}$ beams. These measurements were made with the wedge oriented in the inplane direction. It was previously verified that there were no significant differences in open field beam profile in the inplane and crossplane directions. Measurements of the open field beam profile made with the diode-array in the crossplane and inplane directions differed by a maximum of $1.5 \%$ within the umbra region of a $20 \times 20 \mathrm{~cm}^{2}$ field.

EPID images were acquired of the same wedged fields. The EPID was the aS500 amorphous silicon detector with the IDU11 detector and the R-Arm type support arm. The EPID was positioned at $105 \mathrm{~cm}$ from the source due to detector positioning constraints. Added buildup of $0.5 \mathrm{~cm}$ and $2 \mathrm{~cm}$ water equivalent material were placed on the EPID protective cover to give $\mathrm{d}_{\max }$ buildup including the inherent EPID buildup of the $1 \mathrm{~mm}$ copper plate (Greer and Popescu 2003). The EPID was flood-field (FF) calibrated at this distance with a $40 \times 30 \mathrm{~cm}^{2}$ field. The collimator was rotated to $90^{\circ}$ so that the wedge was oriented in the crossplane direction. Both Y1-in and Y2-out wedges were recorded. The EPID was operated using the frame-averaging dosimetry mode where frames are continuously acquired during acquisition. All irradiations were 150 monitor units (MU) with a dose-rate of $300 \mathrm{MU} / \mathrm{min}$.

The horns inherent in large open fields are not reported by the EPID due to the FF calibration technique. All acquired EPID images are divided by the FF image which is an image of a large field used to correct pixel sensitivity or gain differences. As the acquired EPID image and the FF image both contain the beam profile, these profiles cancel and the profile is removed from the image. To obtain the beam profile from the EPID image, a recently reported EPID dosimetry method was utilized (Greer 2005). The automatically applied FF calibration image was removed by multiplying the FF-corrected EPID image by the floodfield. The EPID image was then corrected for pixel sensitivity variations using a pixel sensitivity correction matrix. This yields the beam profile measured by the EPID if all pixels have the same gain and hence contains the differential energy response of the EPID with offaxis distance. To remove the energy response, the EPID image was corrected with a measured function that describes the radially-dependent EPID response relative to ion-chamber. The results from this method have also been compared to the conventional EPID dosimetry method of multiplying the FF-corrected EPID image by a beam profile matrix obtained from a profile measurement at $d_{\max }$ depth in water with an ion-chamber. Under standardized conditions (EPID position $=$ FF calibration position) these methods produce very similar EPID profiles. However it should be noted that the FF calibration could potentially remove changes in beam and hence EDW profile. If the conventional FF calibration with beam profile correction method is used, care should be taken to verify that the beam profile has not 
changed before FF calibration of the EPID is performed. The comparison of beam profiles recorded by the EPID and the diode-array were performed in MATLAB. Software was written that loads the EPID image, performs the above corrections, and then loads the diodearray results for comparison. To derive the ratio of the two measurements the diode-array results were interpolated to the same resolution as the EPID.

To ensure that diode-array measurements were equivalent to ion-chamber, measurements were made of a $20 \times 20 \mathrm{~cm}^{2} 60^{\circ}$ wedge oriented in the crossplane direction. A $0.6 \mathrm{cc}$ ionchamber was positioned at $5 \mathrm{~cm}$ depth at $100 \mathrm{~cm}$ source-axis distance in solid water at $2.5 \mathrm{~cm}$ intervals to $\pm 7.5 \mathrm{~cm}$ from the central axis and smaller intervals $(0.7-1.2 \mathrm{~cm})$ thereafter. Three readings were made at each position and averaged. Wedge profiles with the diode-array were then obtained with $5 \mathrm{~cm}$ solid water buildup on the array and a source-detector distance of $100 \mathrm{~cm}$. While these setup conditions are slightly different to the above $d_{\max }$ measurements, agreement of the diode-array and ion-chamber measurements at $\mathrm{d}_{\max }$ for an open $20 \times 20 \mathrm{~cm}^{2}$ field was found to be within $0.6 \%$.

\subsection{EPID profile dependence on wedge direction and EPID position}

To ensure that there was no directional dependence of EPID response in the crossplane direction for the same wedge profile, firstly the Y1-in wedge was delivered with the wedge oriented in the crossplane direction with collimator angles of 90 and 270 degrees, and the EPID results compared. The EPID was positioned at $105 \mathrm{~cm}$ from the source as above. To then ensure that the Y1-in and Y2-out wedge profiles were equivalent, the Y1-in and Y2-out wedges were delivered in this direction with the collimator set to 270 degrees. It was not possible to reproduce these tests in the inplane direction as the collimator can not be positioned at 180 degrees, this being beyond the range of collimator motion.

EPID images were then acquired for both Y1-in and Y2-out wedges with the collimator angle set to $0^{\circ}$ for the wedge oriented in the inplane direction. The EPID support arm components are non-uniform in the inplane direction and this could result in differences in dosimetric profiles due to backscattered radiation. These profiles were compared to each other and also to the previous results for the wedge oriented in the crossplane direction. The profiles were rotated when necessary to compare.

The EPID was also positioned at $140 \mathrm{~cm}$ from the source and FF-calibrated at this position with a $23 \times 28 \mathrm{~cm}^{2}$ field. The EPID EDW profile measurements were then repeated at this position with the wedge orientation in both crossplane and inplane directions to determine whether the results differed from the $105 \mathrm{~cm}$ profiles. The diode-array measurements were 
also repeated at this distance to ensure that there was no change in profile from the $100 \mathrm{~cm}$ distance. For all acquired EPID profiles the results were scaled to $100 \mathrm{~cm}$ from the source for comparison to the diode-array measurements.

\subsection{EPID EDW Wedge factor}

The wedge factors derived from EPID images of EDW fields were compared to those obtained from ion-chamber measurements for both beam energies. The wedge factor was obtained from the ratio of the mean pixel response in the central $9 \times 9$ pixel region of a 60 degree $10 \times 10 \mathrm{~cm}^{2}$ wedged field image and an open $10 \times 10 \mathrm{~cm}^{2}$ field image. The EPID was positioned at $105 \mathrm{~cm}$ from the source and an additional $4 \mathrm{~cm}$ of solid water buildup was placed on the EPID cover to give an effective depth of approximately $5 \mathrm{~cm}$ to the EPID sensitive layer. The integrated pixel response from each image was obtained by multiplication of the pixel values by the number of frames acquired. Three EPID images were acquired for the open and wedged fields respectively and averaged at each session. The EPID measurements were repeated for three sessions at weekly intervals. The ion-chamber measurements were performed with a $0.6 \mathrm{cc}$ Farmer chamber at a depth of $10 \mathrm{~cm}$ in water, and a source-axis distance of $100 \mathrm{~cm}$ for treatment planning system commissioning. Weekly measurements are also performed at a depth of $5 \mathrm{~cm}$ in plastic with a Holt chamber, and a source-axis distance of $100 \mathrm{~cm}$. The average of these measurements over a 7 month interval was within $0.1 \%$ of the $10 \mathrm{~cm}$ depth $0.6 \mathrm{cc}$ ion-chamber wedge factors.

\subsection{EPID long-term stability for EDW measurement}

The long-term stability of the EPID profile measurements was investigated for both central axis and beam profile response with a weekly reference setup over a seven month interval for both 6 and $18 \mathrm{MV}$ energies. The EPID dose response was recorded with three images of a $10 \times 10 \mathrm{~cm}^{2}$ open field. Measurement of EDW profiles were made with images of a $60^{\circ}$ EDW field oriented in the inplane direction for a $20 \times 20 \mathrm{~cm}^{2}$ field size. The EPID was positioned at $105 \mathrm{~cm}$ and the buildup for each measurement was $2 \mathrm{~cm}$ added solid water on the EPID.

The EPID dose response was obtained from the mean pixel value in the central $9 \times 9$ pixel region multiplied by the number of frames for each image. This was averaged for the three $10 \times 10 \mathrm{~cm}^{2}$ images. At each imaging session the dose output from the linear accelerator was measured with an ion-chamber constancy set-up for both energies. The ion-chamber was a Holt chamber embedded within a plastic block. All readings were temperature and pressure corrected. 
The EPID normalized profiles from each weekly measurement were compared. A single pixel profile for each image along the central axis in the wedged direction was obtained. These were first aligned using a maximum gradient search, and the standard deviation of the corresponding pixel values in each image calculated. The shifts were a maximum of 4 pixels or approximately $3 \mathrm{~mm}$. The images have a defective pixel map correction applied that replaces defective pixels with the average of the surrounding pixels, and these do not influence the results. The results were analyzed for the central umbral region to within $0.5 \mathrm{~cm}$ of the $50 \%$ penumbral value. The consistency of the EDW profile was also measured monthly with the diode-array as part of the regular quality assurance program for both energies.

The stability of EPID wedge-factor measurements was also assessed over this time period using the above images for both energies. The EPID wedge factor was obtained as described above using the central pixel region of the $20 \times 20 \mathrm{~cm}^{2}$ wedged field, and the $20 \times 20 \mathrm{~cm}^{2}$ open field. The EPID pixel location corresponding to the beam central axis was first determined from the open field before the pixel values were obtained. The $50 \%$ edges were found from interpolating the pixel values either side of the $50 \%$ value. The mid-point was then taken to be the central axis pixel location. The standard deviation of the EPID wedge factor was compared to the standard deviation of weekly ion-chamber measurements of EDW wedge factor over a seven month time interval.

\subsection{Correction of EPID profiles}

The correction of EPID profiles was investigated. A ratio of EDW profiles in water and EPID was derived. A treatment planning system (Pinnacle, Version 6.2b, Phillips Medical Systems, Madison, USA) was used to produce a planar dose map of a $20 \times 20 \mathrm{~cm}^{2}$ field with a $60^{\circ} \mathrm{EDW}$ at $6 \mathrm{MV}$. The dose calculation grid used was $0.2 \mathrm{~cm}$. The SSD was $100 \mathrm{~cm}$, with the planar dose calculated at $1.5 \mathrm{~cm}$ depth. This dose matrix was then interpolated to give samples at the same sampling interval as the EPID pixel map. The planning system dose was used as it could be produced at a much higher resolution than diode-array profiles. The planning system profile was compared to diode-array and the results differed by a maximum of $1 \%$ at the highdose end of the wedge. The EPID EDW (crossplane) image was divided by the TPS calculation to derive a two-dimensional ratio matrix. A profile of this ratio in the wedged direction along the central axis was produced. This was truncated to the region from $0.5 \mathrm{~cm}$ within the penumbra and a quadratic function fitted to this using interactive curve fitting software. EPID profiles of EDW corrected with this function were then compared to diodearray results for both $60^{\circ}$ and $30^{\circ}$ wedge angles for both 6 and $18 \mathrm{MV}$ energies. 


\section{Results}

\subsection{EPID EDW Profile measurement}

Figure 1 shows the EPID EDW crossplane profiles compared to diode-array profiles for the wedged $20 \times 20 \mathrm{~cm}^{2}$ fields normalized to central axis. The ratio of the two profiles is also shown as $1-A / B \times 100 \%$, where $A$ is the EPID profile and $B$ is the diode-array profile. To determine the ratio of the two measurements, the diode-array results were interpolated to give a profile with the same sampling interval as the EPID measurements. The EPID profiles are lower at the high and low-dose ends of the wedge by up to $5 \%$. The results for the $10 \times 10 \mathrm{~cm}^{2}$ fields were similar and are not shown. The agreement between the Y1-in and Y2-out EPID wedge profiles was very close as discussed in the next section.

The diode-array profile for the $60^{\circ}$ wedge at 6 and $18 \mathrm{MV}$ agreed within $2 \%$ of point-ion chamber measurements made with a 0.6 cc ion-chamber. The ion-chamber measurements were slightly lower in the high-dose end of the wedge, which is to be expected given the large physical size of the ion-chamber and the large dose gradient within this region. Therefore the diode-array measurements provide an accurate representation of the actual EDW wedge profile for comparison to EPID measurements.

\subsection{EPID profile dependence on wedge direction and EPID position}

To ensure that the Y1-in and Y2-out wedges were equivalent, firstly the Y1-in wedge was delivered in the crossplane direction with collimator angles of 90 and 270 degrees. These (following rotation and alignment of the profiles) were in very close agreement (within $0.5 \%$ ) showing that there was no directional dependence of EPID response in the crossplane direction for the same wedge profile. Then the Y1-in and Y2-out wedges were delivered in this direction with the collimator set to 270 degrees. These were within $1 \%$ maximum difference showing that the Y1-in and Y2-out wedges deliver almost identical beam profiles. It was not possible to reproduce these tests in the inplane direction as the collimator can not be positioned at 180 degrees, this being beyond the range of collimator motion.

The comparison of EPID profiles for Y1-in and Y2-out wedges oriented in the inplane direction is shown in Figure 2(a). The profiles are shown with the flood-field correction removed, along with the flood-field profile for comparison. These clearly show the increase in dose at the superior end of the wedge in the region where the high density support arm components that can backscatter radiation are located. These components are mainly in the region of the EPID closer to the gantry. 
In Figure 2(b) the flood-field corrected, and aligned profiles are shown. These are slightly lower in magnitude than the Figure 1 results as these are the flood-field corrected images reported by the vendors software, without any additional corrections and therefore do not contain the open beam profile. The profiles for the wedge oriented in the inplane direction differ by up to $4 \%$ at the wedge high-dose end.

For the uncorrected images the dose for the Y1-in wedge at the wedge high-dose end is higher than the Y2-out wedge high-dose end as seen in Figure 2(a). When the flood-field correction is applied this reverses, as seen in Figure 2(b). This is due to the fact that the backscatter in the region of the flood-field corresponding to the Y1-in wedge high-dose end is greater for the large flood-field than for the wedged field. This reduces the Y1-in wedge high-dose values in the corrected image. For the Y2-out wedge high-dose region the backscatter component is less and so the flood-field correction has less impact on the dose. A similar effect is seen with small field open beam profiles where there is little backscatter and the flood-field correction produces a slope in the profiles with lower dose in the superior direction.

The comparison of EPID profiles for the Y1-in wedge oriented in the (a) crossplane and (b) inplane directions measured at $105 \mathrm{~cm}$ and $140 \mathrm{~cm}$ from the source are shown in Figure 3. The results have been scaled to $100 \mathrm{~cm}$ for comparison. These show that for the wedge oriented in the crossplane direction the EDW profiles are consistent with detector distance, however for the wedge oriented in the inplane direction the results differ, even though the EPID was flood-field calibrated at each distance. The change in position within the beam of the backscatter components is responsible for this difference. The beam broadens due to divergence, however the support arm components remain fixed with respect to the beam central axis. Therefore the EPID EDW profiles measured for the wedge oriented in the inplane direction are dependent on EPID distance from the source.

\subsection{EPID EDW Wedge factor}

The results for the EPID wedge factor measurements are given in Table 1. These were all within $0.6 \%$ of the ion-chamber reference wedge factors for both energies. The maximum deviation of the weekly EPID measurements was $0.9 \%$. At each weekly session where three EPID images of the open field and the wedged field respectively were averaged, the standard deviation of the three measurements in each case was within $0.11 \%$. 


\subsection{EPID long-term stability for EDW measurement}

The stability of the EPID dose response is shown in Figure 4 for the central axis of the $10 \times 10$ $\mathrm{cm}^{2}$ fields. Each data point represents the average of three EPID images. The response varied by $0.4 \%$ standard deviation for $6 \mathrm{MV}$ and $0.3 \%$ standard deviation for $18 \mathrm{MV}$ over the 7 month interval. These results are corrected for the dose output change of the linear accelerator, measured at each weekly imaging session.

The variation in EPID EDW normalized profiles for the $60^{\circ}$ wedge for the seven month period for each pixel was a maximum $0.3 \%$ standard deviation for both energies. There was one flood-field calibration of the EPID made during this time inverval. The EPID measurements of EDW wedge factor varied by $0.3 \%$ ( 1 standard deviation) with a maximum deviation of $1 \%$ over the seven month interval which is less than the variation of ion-chamber measurements over this time of $0.6 \%$ ( 1 standard deviation). It was found to be necessary to first determine the pixel corresponding to the beam central axis from the open image as the EPID detector position can vary over time.

\subsection{Correction of EPID profiles}

The quadratic function that was derived to correct the EPID measurements was $y=0.00062312 x^{2}-0.00037805 x+1$ where $x$ is the distance in cm scaled to isocentre. The result for the corrected EPID profile for the $60^{\circ}$ wedge at $6 \mathrm{MV}$ is shown in Figure 5(a) compared to diode-array measurements along with the quadratic function. The correction that was derived from the $60^{\circ}$ wedge at $6 \mathrm{MV}$ energy was also applied to the $30^{\circ}$ wedge at $6 \mathrm{MV}$ with the result shown in Figure 5(b) and the $30^{\circ}$ and $60^{\circ}$ wedges at $18 \mathrm{MV}$, the latter result shown in Figure 5(c). These results show that a simple analytical function can be used to obtain accurate EDW dose measurements with the EPID over a range of wedge angles and energies.

\section{Discussion}

The measurement of EDW profile with the EPID is rapid with virtually no experimental setup time compared to the diode-array. The routine assessment of EDW as well as open field profile is now performed in our department with the EPID. A QA patient has been setup to deliver the fields on the linear accelerator and acquire the EPID images. These are exported from the vendors software (Varis Version 7, Review Task) and assessed in a MATLAB software module. The module loads a baseline EPID image, and the current QA image, aligns the two and compares the central axis profile both visually and quantitatively. The alignment is based on the central axis pixel coordinate that is derived for each imaging session from a $10 \times 10 \mathrm{~cm}^{2}$ image. It should be noted that the vendor recommends a delay of 10-15 s between 
initiating image acquisition and beam-on when acquiring images outside of the Varis system. This is because the IAS2 software may not be ready to acquire data even when the software reports that it is ready. Marked degradation in the reproducibility of images acquired in the same session was found when this delay was not observed.

The vendor can supply an IMRT verification "dosimetry" software module designed to compare a reference (predicted) EPID image generated by the Eclipse planning system with an acquired EPID image. This could potentially be used for EDW quality assurance, as an acquired baseline EPID image can be saved to an ASCII format and re-imported as the reference dose image. However this software is currently designed for absolute dose comparison, and an image normalization feature is not available. The ability to normalize the reference and currently acquired EDW images to the central axis, would enable this software to be used for routine EDW analysis.

The comparison of EPID profiles for EDW with conventional diode-array measurement show a lowering or "rounding" of the EPID profiles near the field edges. The EPID profiles have been corrected for the spectral over-response of the EPID relative to ion-chamber with offaxis distance. This effect may be due to optical scatter within the phosphor layer. El-Mohri et al. (1999) compared profiles from a prototype flat-panel EPID with and without the phosphor layer present. They found that with the phosphor layer removed and the EPID operating in "direct detection mode" that the EPID penumbra matched closely to ion-chamber measurements. With the phosphor layer present the EPID profiles were more rounded or blurred than the ion-chamber. McCurdy et al. (2001) also postulated that optical glare would explain the lack of inverse square fall-off of dose in the tail region of EPID profiles measured at different source to EPID distances. They incorporated a glare kernel into their EPID dose prediction model to improve agreement with measured images. Kirkby et al. (2005a) found that by incorporating optical scatter in their model of the EPID that they obtained better agreement of deconvolved EPID fluence with measurements. Potentially this effect could be accounted for by a deconvolution of an EPID scatter kernel followed by a convolution with a water dose kernel (Warkentin et al 2003). This however would make the calculation of the dose profile difficult for most users of the EPID who do not have access to these methods.

For routine QA measurements correction of the EPID profile is not required, as the reproducibility of the EDW profiles can be monitored. However, it may be desirable for initial wedge commissioning, or annual quality assurance tests to measure the actual wedge profile. We chose therefore to investigate whether a simple analytical correction function could be applied to the EPID measurements to yield EDW dose profiles in good agreement 
with ion-chamber or diode-array measurements. The correction function would ideally be measurement based, however we used the ratio of EPID and treatment planning system dose planes as the planning system dose could be produced at a much higher resolution than diodearray profiles. An alternative would be to interpolate the diode-array profiles. This method was found to be very effective, and is simple and easy to implement. A single function works well for a range of wedge angles and energies. While this method can not correct differences in penumbra of EPID and ion-chamber/diode measurements, this is of minor concern for routine QA measurement. Routine diode-array measurements have coarse resolution and do not accurately record beam penumbra. In effect this method may correct for the difference in profile due to the difference in long-range scatter kernels of the EPID and water.

The EPID profiles for the wedge oriented in the inplane direction were found to vary with wedge direction and source to EPID distance. There was no variation in these profiles when measured with diode-array. This is due to the effect of non-uniform backscattered radiation from the EPID support arm components beneath the detector. At the greater distance the head scatter may also differ and since this scatter is of lower energy, the energy response of the EPID could vary. To correct for these effects would be difficult. The profiles are consistent for the wedge oriented in the crossplane direction, and these can be measured by rotating the collimator to 90 degrees for the EDW measurements. An important point to note is that the EPID routine measurements should be performed with standardized conditions to avoid differences occurring due to the backscatter.

The reproducibility measurements show that the EPID is a reliable dosimeter and can certainly be used for relative dose measurements, and potentially quantitative dose measurements. A possibility exists to measure absolute dose EDW profiles as a check of both EDW profile and wedge factor simultaneously.

\section{Conclusions}

The EPID measurements of EDW profiles and wedge-factors were investigated by comparison to conventional ion-chamber and diode-array measurements. EPID measurements were found to exhibit a dependence on wedge direction and EPID position due to backscatter from the EPID housing. A single empirical correction function was developed that produces EPID wedge profiles that correspond to diode or ion-chamber measured profiles for all wedge angles and energies investigated. Due to the reproducibility of the EPID measured EDW profiles the device is highly suited to regular measurement of EDW. This can be performed in significantly less time than conventional methods. 


\section{References}

Budgell G, Zhang Q, Trouncer R and Mackay R 2005 Improving IMRT quality control efficiency using an amorphous silicon electronic portal imager Med. Phys. 32 3267-3278

Chang J and Ling CC 2003 Using the frame averaging of aS500 EPID for IMRT verification J. Clin. Appl. Med. Phys. 4 287-299

El-Mohri Y, Antonuk L, Yorkston J, Jee K, Maolinbay M, Lam K and Siewerdsen J 1999 Relative dosimetry using active matrix flat-panel imager (AMFPI) technology Med. Phys. 26 $1530-1541$

Greer P and Popescu C 2003 Dosimetric properties of an amorphous silicon electronic portal imaging device for verification of dynamic intensity modulated radiation therapy Med. Phys. 30 1618-1627

Greer P 2005 Correction of pixel sensitivity variation and off-axis response for amorphous silicon EPID dosimetry Med. Phys. 32 3558-3568

Grein E, Lee R, Luchka K 2002 An investigation of a new amorphous silicon electronic portal imaging device for transit dosimetry Med. Phys 29 2262-2267

Kirkby C and Sloboda R 2005a Comprehensive Monte Carlo calculation of the point spread function for a commercial a-Si EPID Med. Phys. 32 1115-1127

Kirkby C and Sloboda R 2005b Consequences of the spectral response of an a-Si EPID and implications for dosimetric calibration Med. Phys. 32 2649-2658

Ko L, Kim J and Siebers J 2004 Investigation of the optimal backscatter for an aSi electronic portal imaging device Phys. Med. Biol. 49 1723-1738

Louwe R, McDermott L, Sonke J-J, Tielenburg R, Wendling M, van Herk M and Mijnheer B 2004 The long-term stability of amorphous silicon flat panel imaging devices for dosimetry purposes Med. Phys. 31 2989-2995

McCurdy B, Luchka K and Pistorius S 2001 Dosimetric investigation and portal dose image prediction using an amorphous silicon electronic portal imaging device Med. Phys. 28 911924

Munro P, Bouius D 1998 X-ray quantum limited portal imaging using amorphous silicon flatpanel arrays Med. Phys. 25 689-702

Siebers J, Kim J, Ko L, Keall P and Mohan R 2004 Monte-Carlo computation of dosimetric amorphous silicon electronic portal images Med. Phys. 31 2135-2146

van Esch A, Depuydt T and Huyskens D 2004 The use of an aSi-based EPID for routine absolute dosimetric per-treatment verification of dynamic IMRT fields Radiother. Oncol. 71 223-234

Warkentin B, Steciw S, Rathee S and Fallone B 2003,Dosimetric IMRT verification with a flat-panel EPID Med. Phys. 30 3143-3155 


\section{Figures}

Figure 1 EPID EDW profiles compared to diode-array measurements for the $60^{\circ}$ and $30^{\circ}$ wedges for (a) $6 \mathrm{MV}$, and b) $18 \mathrm{MV}$. The ratio in percent of the two profiles is also shown as $(1-A / B) \times 100 \%$, where $A$ is the EPID profile and $B$ is the diode-array profile.

Figure 2 EPID EDW profiles with wedge direction. The comparison of EPID profiles for Y1in and Y2-out wedges is shown for the inplane direction for: a) flood-field correction removed, along with the flood-field image profile, and b) flood-field corrected, and aligned profiles. The negative axis is toward the gun and the positive toward the target.

Figure 3 Comparison of EPID profiles with EPID distance from the source for the $6 \mathrm{MV}$ beam. Profiles for the Y1-in wedge measured at $105 \mathrm{~cm}$ and $140 \mathrm{~cm}$ from the source are shown for (a) crossplane and (b) inplane directions.

Figure 4 Stability of the EPID central axis absolute dose response over the seven month interval for both 6 and $18 \mathrm{MV}$ beam energies. The EPID dose respone is shown relative to ion-chamber measurements.

Figure 5 The EPID profiles corrected with the empirically derived quadratic function compared to diode-array measurements are shown for a) $60^{\circ}$ wedge and b) $30^{\circ}$ wedge at 6 $\mathrm{MV}$, and b) $60^{\circ}$ wedge at $18 \mathrm{MV}$. 


\section{Tables}

Table 1 EDW measurements of wedge factor. The wedge factor derived from three weekly measurements of the central $9 \times 9$ pixel region of a 60 degree $10 \times 10 \mathrm{~cm}^{2}$ wedged field image and an open $10 \times 10 \mathrm{~cm}^{2}$ field image are compared to ion-chamber measurements. 


\section{Figures}
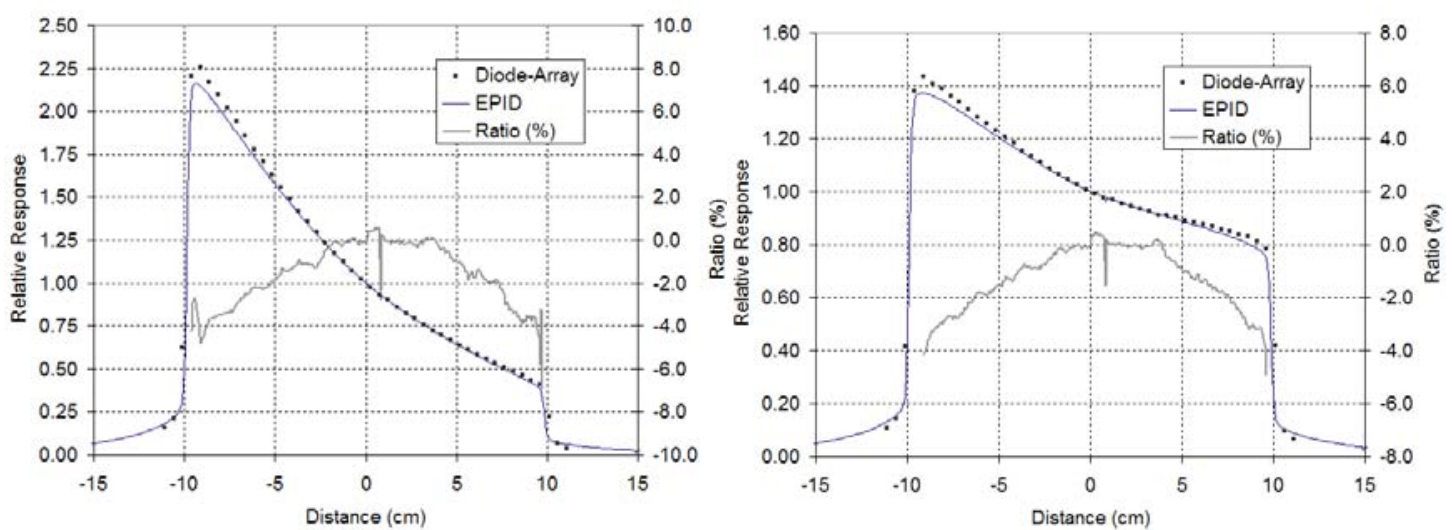

(a)
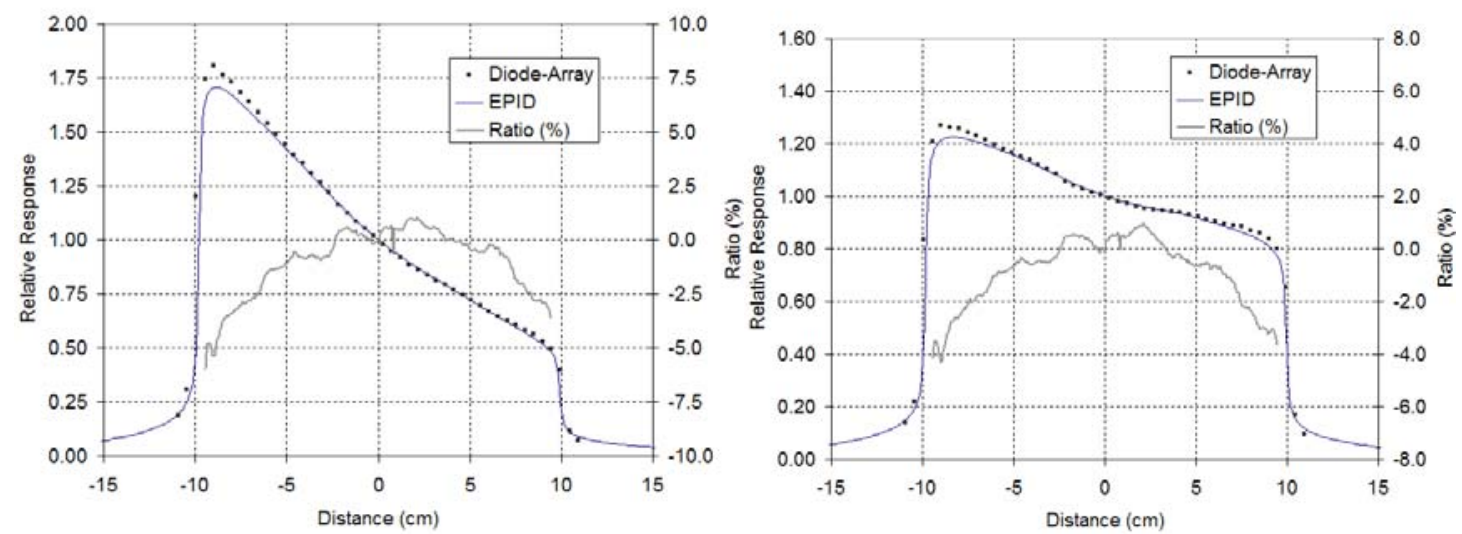

(b)

Figure 1 EPID EDW profiles compared to diode-array measurements for the $60^{\circ}$ and $30^{\circ}$ wedges for (a) $6 \mathrm{MV}$, and b) $18 \mathrm{MV}$. The ratio in percent of the two profiles is also shown as $(1-A / B) \times 100 \%$, where $A$ is the EPID profile and $B$ is the diode-array profile. 


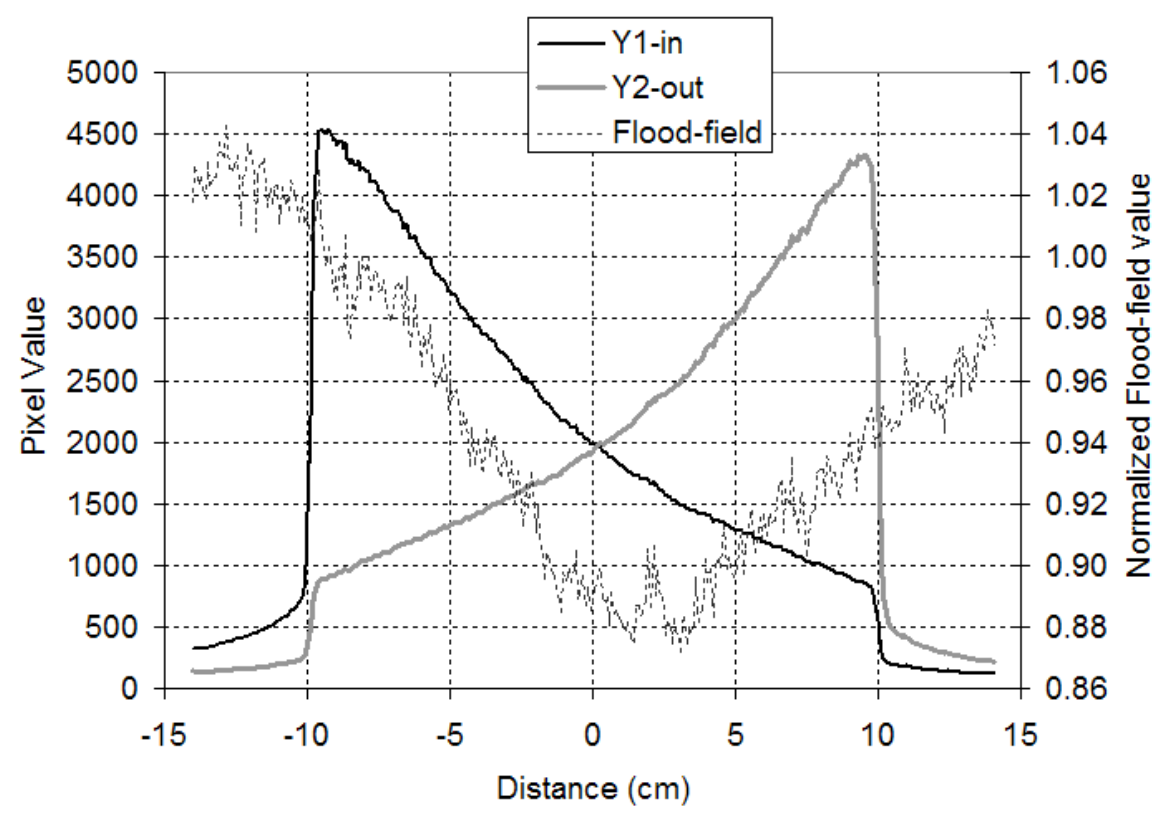

(a)

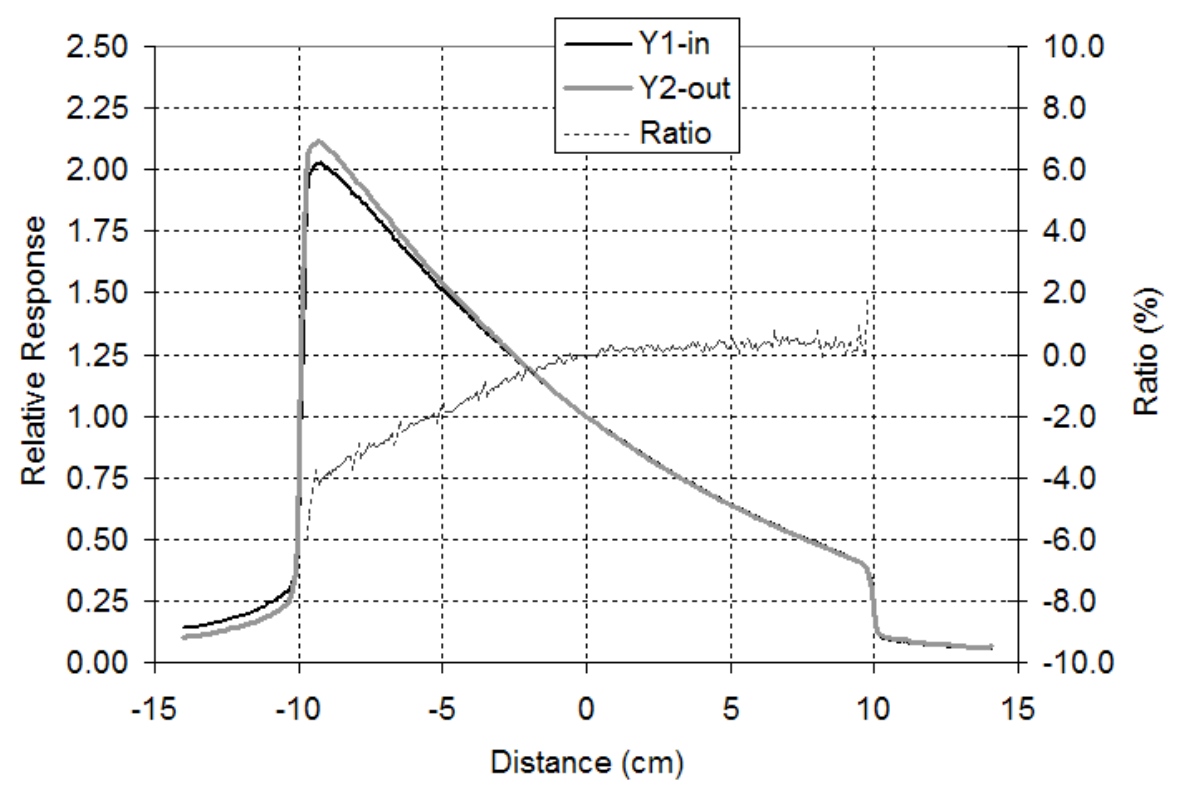

(b)

Figure 2 EPID EDW profiles with wedge direction. The comparison of EPID profiles for Y1-in and Y2-out wedges is shown for the inplane direction for: a) flood-field correction removed, along with the flood-field image profile, and b) flood-field corrected, and aligned profiles. The negative axis is toward the gun and the positive toward the target. 


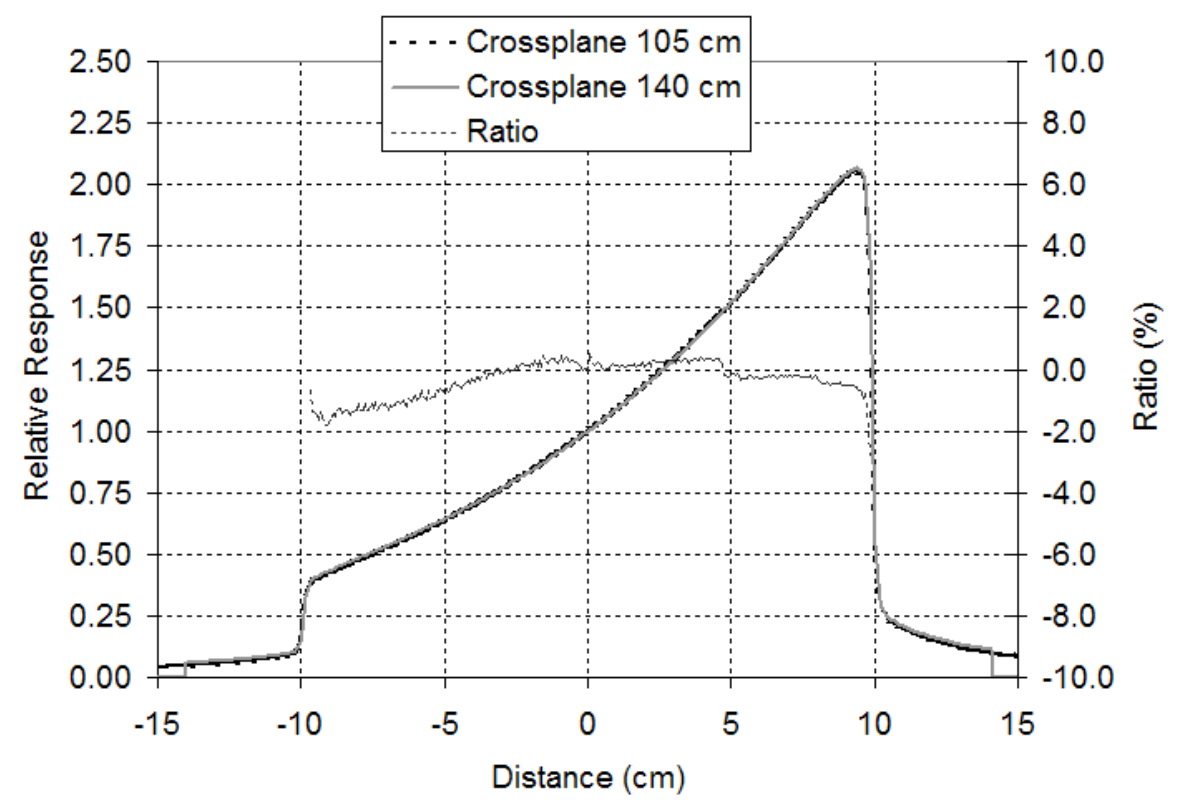

(a)

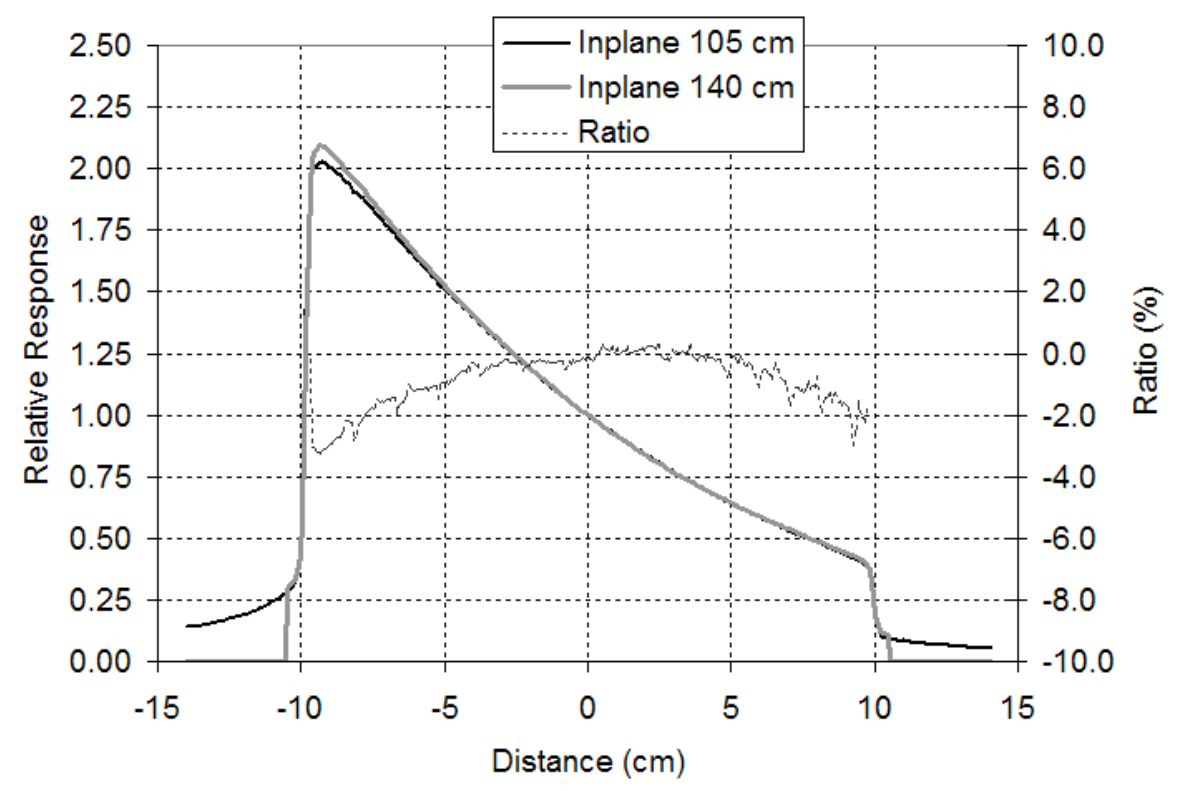

(b)

Figure 3 Comparison of EPID profiles with EPID distance from the source for the 6 MV beam. Profiles for the Y1-in wedge measured at $105 \mathrm{~cm}$ and $140 \mathrm{~cm}$ from the source are shown for (a) crossplane and (b) inplane directions. 


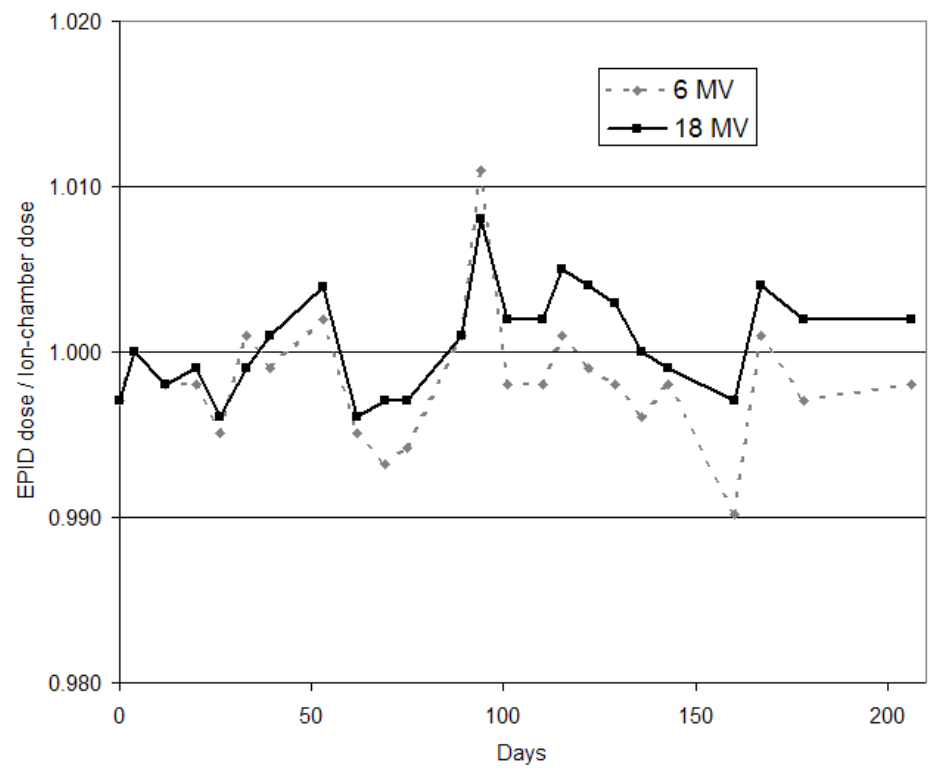

Figure 4 Stability of the EPID central axis absolute dose response over the seven month interval for both 6 and $18 \mathrm{MV}$. The EPID dose respone is shown relative to ion-chamber measurements.

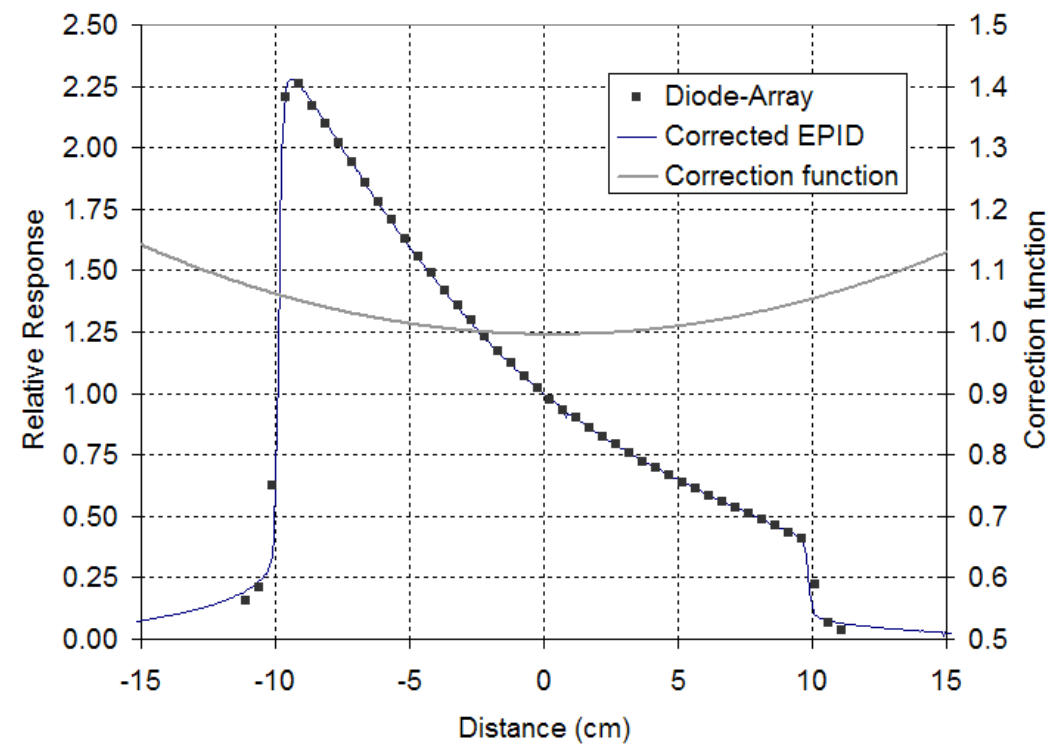

(a) 


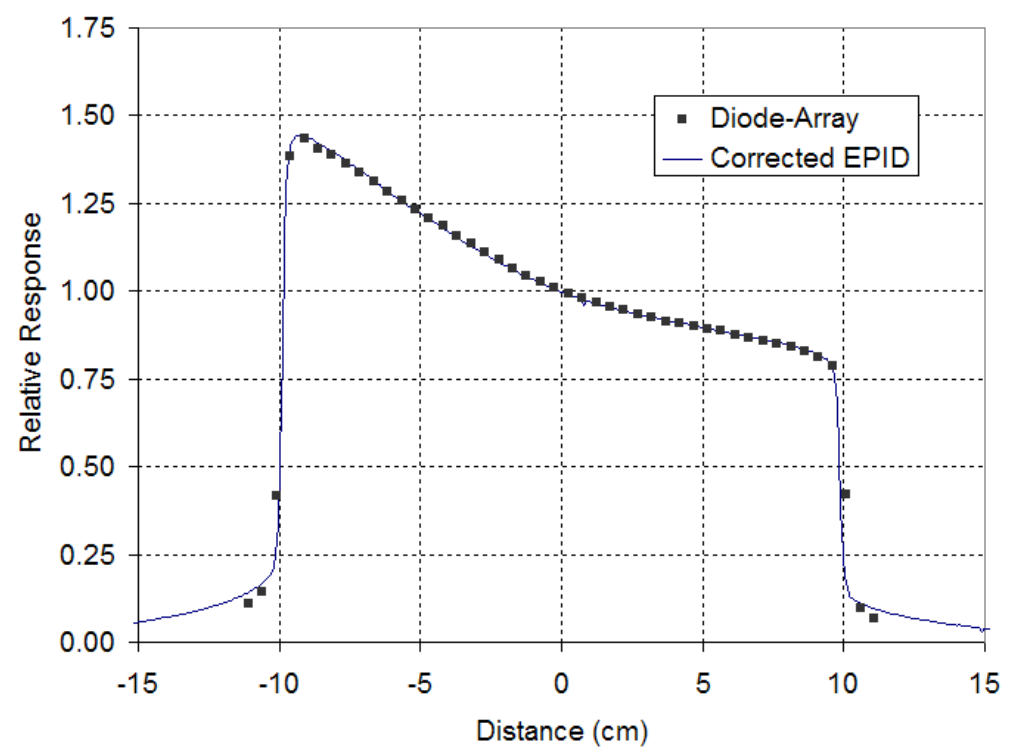

(b)

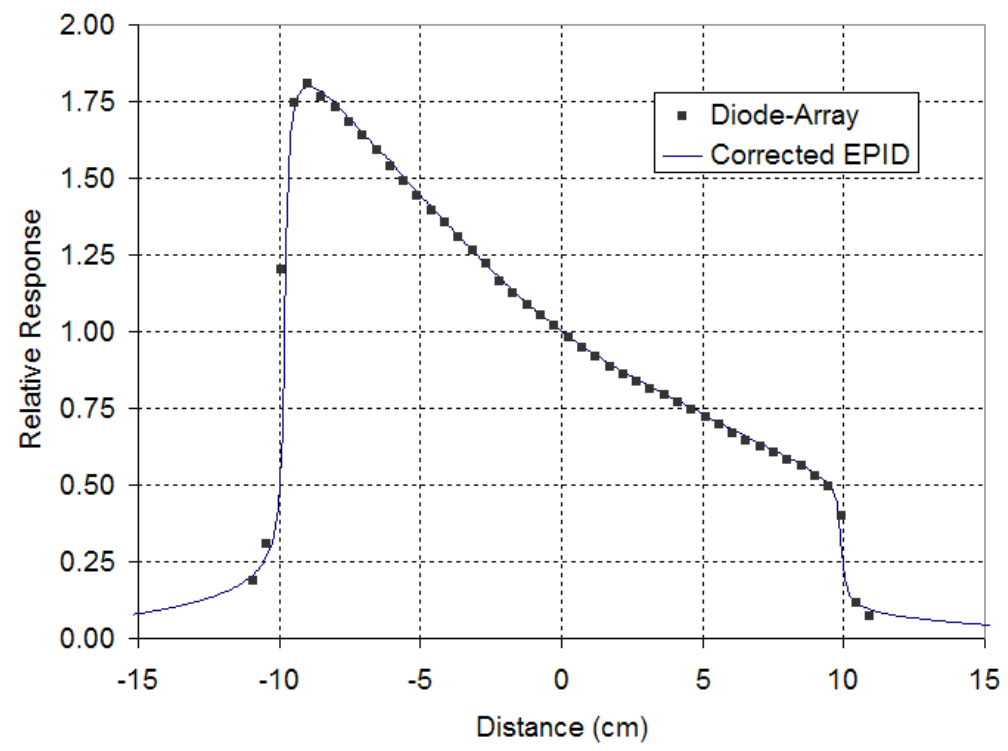

(c)

Figure 5 The EPID profiles corrected with the empirically derived quadratic function compared to diode-array measurements are shown for a) $60^{\circ}$ wedge and b) $30^{\circ}$ wedge at 6 $\mathrm{MV}$, and b) $60^{\circ}$ wedge at $18 \mathrm{MV}$. 


\begin{tabular}{ccccc}
\hline Energy & \multicolumn{5}{c}{ Wedge Factors } \\
\hline & Ion-Chamber & EPID Week 1 & EPID Week 2 & EPID Week 3 \\
$6 \mathrm{MV}$ & 0.659 & 0.663 & 0.657 & 0.660 \\
$18 \mathrm{MV}$ & 0.728 & 0.729 & 0.723 & 0.725 \\
\hline
\end{tabular}

Table 1 EDW measurements of wedge factor. The wedge factor derived from three weekly measurements of the central $9 \times 9$ pixel region of a 60 degree $10 \times 10 \mathrm{~cm}^{2}$ wedged field image and an open $10 \times 10 \mathrm{~cm}^{2}$ field image are compared to ion-chamber measurements. 